\title{
Characterization of acoustic diffusion using refracto-vibrometry
}

Published in Journal of Sound and Vibration 393 (2017) 70-76

Submitted in revised form 15/12/16. Published online 20/12/16

This is a post-review proof.

Richard Jackett ${ }^{\mathrm{a})}$

Ben Piper ${ }^{\text {) }}$

Richard J. Hughes ${ }^{\text {c) }}$

a,b) National Physical Laboratory, Hampton Rd, Teddington, TW11 0LW, United Kingdom

c) Acoustics Research Centre, University of Salford, Salford, M5 4WT, United Kingdom

Refracto-vibrometry is a relatively new measurement technique that is sensitive to variations in the optical refractive index of a medium caused by changes in acoustic pressure within that medium (the acousto-optic effect). It has so far been employed primarily as a qualitative visualization tool for airborne sound propagation because determining sound pressure level at a point using the technique is difficult and inefficient. Instead, the authors propose that this optical technique is well suited for determining dimensionless quantities, such as coefficients describing scattering uniformity from a surface. A new measurement and analysis process relying on refractovibrometry has been developed and used to determine acoustic diffusion coefficients through purely optical means for the first time. A quadratic residue diffuser is used as an arbitrary test surface, and refracto-vibrometry measurements of its polar response have been performed and results compared to a boundary element model. The benefits and limitations of the optical method over the traditional microphone-based approach are discussed.

Keywords: refracto-vibrometry, acousto-optic effect, diffusion coefficient, scattering coefficient, optical measurement technique
a) email: richardjackett@gmail.com (corresponding author)
b) email: ben.piper@npl.co.uk
c) email: r.j.hughes@salford.ac.uk 


\section{INTRODUCTION}

The acoustic quality of concert halls, exhibition centres, recording studios, and indeed any enclosed space, is principally defined by their size, shape, and the nature of reflections from the surfaces within that space [1]. Architects and acousticians depend on having accurate information about the absorption, reflection, and scattering properties of surface materials in order to correctly specify and realize their designs [2]. Whilst absorption is often measured in large reverberation chambers, applying statistical assumptions based on an assumed diffuse field, the characterisation of the spatial scattering of surfaces is somewhat more involved. An important property of a surface defined by the latter is the diffusion coefficient - a measure of scattering uniformity with angle [3]. Measuring the scattering properties of surface samples accurately is a laborious and time consuming process because it relies on making a great many measurements with calibrated microphones [3,4] - for diffusion measurements at least 37 microphones are required for simultaneous measurement over a semi-circle, or a bulky goniometer for sequential measurement in $2 \mathrm{D}$ or $3 \mathrm{D}$. There is scope for substantial improvement to the speed and accuracy of sample testing if microphone and goniometer based systems can be replaced by optical equivalents.

The use of light to map the distribution of sound in 2D fields has been in existence for more than 300 years, going back to the first experiments in Schlieren imaging and shadowography [5]. A modern equivalent, refracto-vibrometry (which uses a laser Doppler vibrometer - primarily developed to study vibrating solid surfaces) allows very rapid and precise optical sampling, and may be used to observe sound propagation in optically transparent media via an interaction known as the acousto-optic effect [6]. From the late $20^{\text {th }}$ century refracto-vibrometry has been used to image ultrasonic waves in water [7], and over the last 15 years it has been successfully applied to image sound propagation in air [8]. Two frames showing sound propagation (reflected from a diffuser) constructed using refracto-vibrometry are presented in fig. 1 to illustrate one qualitative application of the technique.
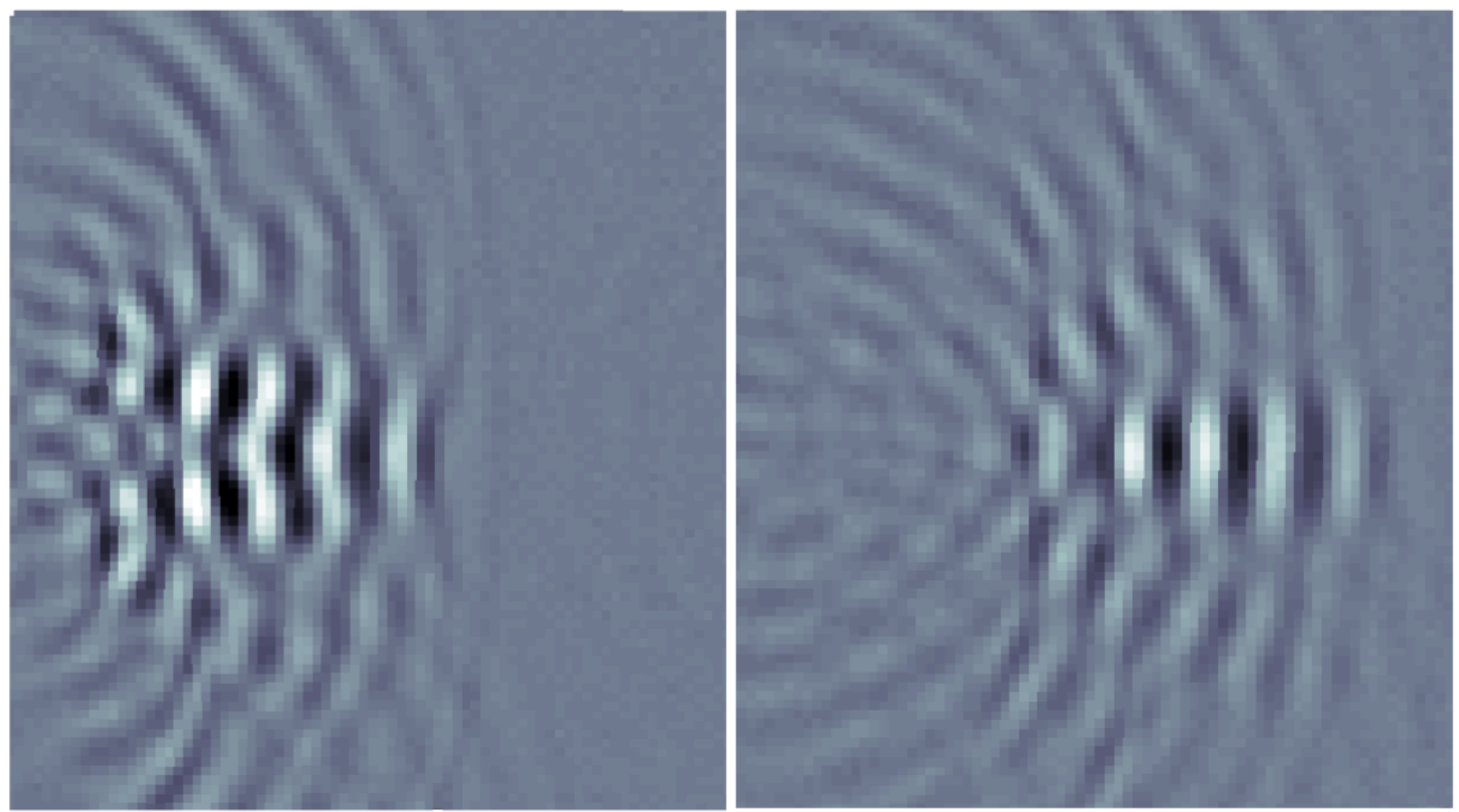
Fig. 1 - Full-field scans of a full scale $(0.6 \mathrm{~m}) \mathrm{N}=7$ QRD diffuser at the left edge of each frame, facing rightwards (not shown) reflecting a $4 \mathrm{kHz}$ tone burst. The two frames are snapshots of the reflected sound field at $2 \mathrm{~ms}$ (left) and $3 \mathrm{~ms}$ (right) after first reflection, each showing an area of about $1 \mathrm{~m} \times 1 \mathrm{~m}$ at $1 \mathrm{~cm}^{2}$ resolution.

The acousto-optic effect describes how the optical refractive index of a medium, $n$, is dependent on the pressure, and is therefore influenced by sound pressure, $p$, in a deterministic way (Eq. 1).

$$
n \cong n_{0}+\frac{n_{0}-1}{\gamma p_{0}} p
$$

Where $n_{0}$ is the static refractive index (close to 1 in air), $p_{0}$ the static pressure, and $\gamma$ the ratio of specific heats. The ratio $p / p_{0}$ and the difference $\left(n_{0}-1\right)$ confirm that this is an incredibly weak effect in air, and therefore high sound pressures are required for it to be observable.

The change to refractive index alters speed of light through that region, which is detected through refracto-vibrometry as an apparent Doppler velocity, $v$. This velocity is proportional to the rate of change of the line integral of acoustic pressure along the laser's path, L, as described by Eq. 2 .

$$
v(t)=\frac{n_{0}-1}{\gamma p_{0} n_{0}} \frac{\mathrm{d}}{\mathrm{d} t}\left(\int_{\mathbf{L}} p(\boldsymbol{x}, t) \mathrm{d} l\right)
$$

A limitation of the technique is immediately apparent: the line integral of acoustic pressure provides no direct way to determine sound pressure at a point, which is the quantity most commonly sought in acoustic measurements. Tomography has been successfully applied [6,9] to resolve this, although this is very time intensive. Alternatively, for situations where there is a-priori knowledge of the sound field, it is possible to use that in combination with refracto-vibrometry to determine sound pressure at a point [10].

The authors propose in this paper that many of the benefits of refracto-vibrometry can also be directly realized by using it to determine normalized quantities, such as the coefficients describing acoustic reflections (absorption, scattering, and diffusion), where an absolute evaluation of sound pressure is not required. To demonstrate this, an experiment has been conducted on a single plane scattering surface, arbitrarily chosen as a 1D length 7 quadratic residue diffuser (QRD) [11] with well depth sequence [ $\left[\begin{array}{lllllll}0 & 1 & 4 & 2 & 2 & 4 & 1\end{array}\right]$, and it will be shown that polar responses and directional diffusion coefficients can be derived directly from the optical data.

\section{METHODOLOGY}

\subsection{General refracto-vibrometry method}

Laser Doppler vibrometry (as opposed to refracto-vibrometry) measures the vibration of a surface by performing interferometry on a beam of laser light reflected off that surface. In refractovibrometry the reflective surface is fixed in place, and it is variations in the refractive index of the air that causes phase changes to be detected through interferometry. Both applications use a laser 
Doppler vibrometer, which is a type of interferometer designed to be sensitive to Doppler shifts of the returning laser light.

In this study, refracto-vibrometry measurements have been made with a scanning laser vibrometer mounted in a fixed position, with the beam directed towards a large stationary retro-reflector opposite in order to reflect the beam back to the detector, and the sound field of interest occupying the intervening space (fig. 2). A single measurement consists of a sampling of apparent Doppler velocity over a given time interval, usually including synchronous averaging over several acoustic stimuli to improve signal-to-noise ratio (SNR). Scans of the sound field are constructed by repeating this process for different positions in 2 dimensions, while maintaining synchronous triggering of the acoustic stimulus with the start of each measurement.

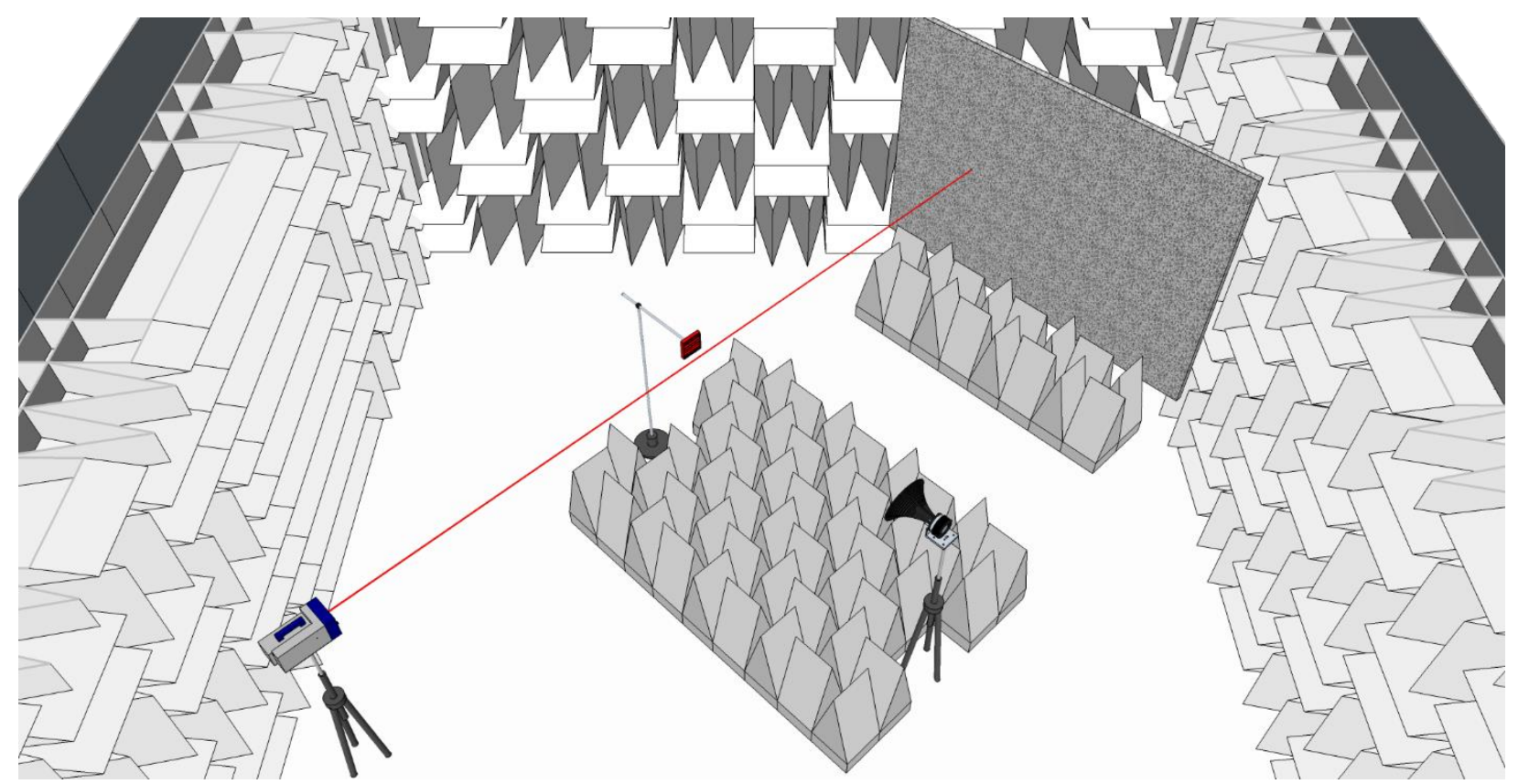

Fig. 2 - The experimental setup in NPL's hemi-anechoic chamber. The PSV-400M is at the bottom left, with its laser beam grazing the diffuser (centre left) and reflecting off the retroreflective curtain (top right). The loudspeaker (centre right) is separated from the diffuser by approximately 2 metres.

NPL's vibrometer scans using rotation rather than translation, which has great practical advantages, but results in a conical projection rather than a parallel projection. By placing the vibrometer 'far' from the sound field of interest, the difference between the two projections is minimised, which is advantageous to interpretation of the data, but requires a very large retroreflector, thus we have employed a retro-reflective curtain to this end.

The geometrical size, shape, and resolution of the 2D scan may be defined in the vibrometer's control software, depending on the application. For example, a regular rectangular grid is suitable for visualisation of a full sound field (fig. 1), while a radial arc of measurement positions is preferable for producing polar plots of sound emission. Once collected, the time series data may be analysed using a software package of choice. 


\subsection{Measurements for determining diffusion coefficients}

The methodology we used to compute the directional and random incidence diffusion coefficients broadly follows that of ISO 17497-2:2012 [3], with the obvious exception that optical measurements are substituted for microphone measurements. In essence, the sound field in front of the test surface is measured position by position in an arc from $+90^{\circ}$ to $-90^{\circ}$ relative to the normal axis, at $5^{\circ}$ resolution, and at a fixed radius from the surface.

This is repeated for several different angles of incidence of the surface to the incoming sound. These measurements contain both the incoming and reflected sound fields. A second set of 'empty field' measurements is made without the surface in place (using identical measurement positions) and subtracted from the first set to remove the incident sound, ideally leaving only the reflections from the test surface. Bandpass filtering of the data is performed, if required, and a time-window is applied to isolate the reflections of interest from any remaining sources of contamination. Finally, an energy summation for each position leads to the production of polar responses for the given frequency and angle of incidence, which are inputs to the diffusion coefficient calculations.

\subsection{Instrumentation}

A Polytec PSV-400M scanning laser vibrometer was set up in a hemi-anechoic chamber with a free working space of $6 \mathrm{~m} \times 6 \mathrm{~m} \times 5 \mathrm{~m}$ high, and supplemented with further sound absorbing polyurethane foam wedges on the floor surrounding the test area (Fig. 2). The vibrometer's laser beam was directed towards a $6 \mathrm{~m}^{2}$ retroreflective curtain suspended normal to the beam in the corner diagonally opposite (approximately $6 \mathrm{~m}$ separation).

A 1:4 scale model $(15 \mathrm{~cm} \times 15 \mathrm{~cm})$ of RPG's QRD-734 diffuser [12], was 3D printed in dense nylon, and mounted such that the horizontal beam passed across its surface. Use of a scale model allows measurements to be taken in the far-field (approximately $50 \mathrm{~cm}$, where over $80 \%$ of the sampling locations fall outside of the specular zone [3]) and also shifts the measurement frequency range up to approximately $1 \mathrm{kHz}$ to $20 \mathrm{kHz}$, where refracto-vibrometry is more sensitive [13], but with the drawback that the scale model reflects $1 / 16^{\text {th }}$ of the acoustic energy of the full-scale diffuser.

A Faital PRO 144 loudspeaker compression driver fitted to a Tractrix LTH142 horn (nominal coverage angle $60^{\circ} \times 50^{\circ}$ ) was placed opposite the diffuser, approximately 2 metres away and with its axis aligned with the normal of the diffuser. This setup is capable of producing a sound pressure level at the diffuser of approximately $110 \mathrm{~dB}$ over most of the measurement frequency range.

\subsection{Source signal and post-processing}

With the setup described, there is approximately $10 \mathrm{~ms}$ between sound emission from the speaker and when the expanding wavefront arrives at and displaces the curtain, which dominates the measurement of Doppler velocity from that moment onwards. Purely acoustic measurements can only be made directly during the first $10 \mathrm{~ms}$, but reflections from the test surface can be recovered over a longer period by subtraction of the empty field measurement; cancelling out the curtain motion to a large extent. 
Several of the acoustic stimulus options from ISO 17497-2:2012 have been trialled. Tone bursts and sine sweeps were found to be effective when employing averaging over many repetitions (typically 200). Impulse-response measurements were also attempted but the available equipment was not capable of producing an impulse of sufficient quality.

Generating tone bursts consisting of approximately four cycles each allowed measurement of reflections for the frequency range $2 \mathrm{kHz}$ to $20 \mathrm{kHz}$ at $1 / 3$-octave spacing. This provided a reasonable signal-to-noise ratio over most of the frequency range, limited by the sensitivity of refracto-vibrometry at low frequency (Eq. 2), and by the sensitivity of the speaker at low and high frequencies. Band-pass filtering improved the signal-to-noise ratio further, before. Finally, a time window (a few ms in duration) was applied to isolate the reflections of interest, and the polar response was calculated.

A swept sine stimulus from $600 \mathrm{~Hz}$ to $22 \mathrm{kHz}$ was also attempted, albeit with limited low frequency resolution. This required use of a time window greater than $10 \mathrm{~ms}$, which therefore included the curtain displacement effect, but empty field subtraction successfully recovered the acoustic reflections from the diffuser, despite them being an order of magnitude smaller than the effect of curtain displacement. Measurement speed was greatly improved over the stepped tone burst approach, from over 2 hours down to 20 minutes. Band-pass filtering and time windowing were employed to separate the reflections into 1/3-octave frequency intervals, and polar responses calculated.

\subsection{Computation of coefficients}

For an anisotropic diffuser, the standard method for measuring the diffusion coefficient [3] specifies measurement in the plane of maximum diffusion in a $180^{\circ}$ arc of constant radius centred on the diffuser. The refracto-vibrometric approach, although it cannot make point measurements in the plane, takes line integrals normal* to the plane, which for this application can be considered to be a centre-weighted projection onto the plane of maximum diffusion. The slight centreweighting arises because the measured Doppler velocity is proportional to the rate of change of the pressure integral (Eq. 2), and is therefore most sensitive when the beam is tangential to the advancing acoustic wavefronts.

Computation of the directional diffusion coefficient, $d_{\theta}$, follows equation 5 of ISO 17497-2:2012 (Eq. 3), with the exception that the rms pressure, $p_{\mathrm{i}}$, at each point $i$ around the arc is necessarily replaced by the rms line integral of pressure along the beam, $P_{\mathrm{i}}$.

$$
d_{\theta}=\frac{\left(\sum_{i=1}^{n} P_{i}\right)^{2}-\sum_{i=1}^{n}\left(P_{i}\right)^{2}}{(n-1) \sum_{i=1}^{n}\left(P_{i}\right)^{2}}
$$

$P_{\mathrm{i}}(t)$, having units of pascal metres, follows directly from Eq. 2, and in practice is found from numerical integration of the apparent Doppler velocity, $v_{\mathrm{i}}$ (Eq. 4). The values for the scalar $P_{\mathrm{i}}$ are

\footnotetext{
* NPL's vibrometer scans using rotation rather than translation, resulting in a conical projection rather than a parallel projection. This is mitigated to some extent by placing the vibrometer 'far' from the test surface, and in any event, all points around the arc are equally affected, further reducing the possibility of a geometrical bias for 1D diffusers.
} 
found by taking the rms of $P_{\mathrm{i}}(t)$ within the fixed time window covering the full reflection, which was previously defined.

$$
P_{i}(t)=\frac{\gamma p_{0} n_{0}}{n_{0}-1} \int v_{i}(t) \mathrm{d} t
$$

The random incidence diffusion coefficient follows from combining directional diffusion coefficients for several different angles of incidence [3].

\section{RESULTS AND ANALYSIS}

To demonstrate the validity of this new measurement approach, the measured results have been compared to numerically modelled results for the same QRD design. A 2D frequency domain Boundary Element Method (BEM) model [14] was used to predict the performance of a single period of the scale model diffuser used for the measurements (note: although a single period would not be applied in practice, this still serves to test the validity of the proposed method). The BEM model uses the Helmholtz Kirchhoff integral equation, expanded using Green's first and second theorems, to formulate the pressure at a point in terms of the incident pressure from a source and a surface integral over the diffuser surface of both pressure and its derivative. This method (excluding small discretisation and numerical precision errors) provides an exact solution to the wave equation, and has been shown previously to provide accurate results for a variety of diffuser types [14]. For simplicity, surfaces were considered to be completely rigid.

Polar plots have been produced to demonstrate the qualitative similarity in spatial scattering between the measurements and the modelled results (fig. 3), with frequencies scaled to the fullsize diffuser. The agreement is in general very good, supporting the viability of refractovibrometric measurements for characterising acoustic reflections. As the directivity becomes more complex at higher frequencies the measurements do not reach the predicted troughs/minima. Several factors have been identified that will cause measurements to diverge from simulated results, presented below in estimated decreasing order of significance:

1. The single frequency model vs. band-passed measurements; the superposition of frequency responses very effectively smooths over minima without greatly affecting maxima (due to the logarithmic nature of SPL).

2. The relatively high noise floor of the measurements (poor SNR), which is particularly evident for the grazing angles of reflection (close to $\pm 90^{\circ}$ ) where the sound pressures are generally lower.

3. The infinite time window of the model vs. the finite time window of the measurements, which affects the bandwidth of tone-bursts, particularly at low frequencies where fewer periods are present. This has a similar effect to factor 1 .

4. The non-perfect nature of the sample and positioning of components compared to the idealised setup of the model.

5. The decreased resolution of measurements $\left(5^{\circ}\right)$ compared to model $\left(1^{\circ}\right)$.

6. The finite sampling area of the measurement (due to the width of the laser beam; also a factor with microphone-based techniques).

7. A 2D model vs. 3D reality, including effects from the conical projection.

Of these, the first two are considered to cause the majority of the discrepancy with the modelled polar responses. 


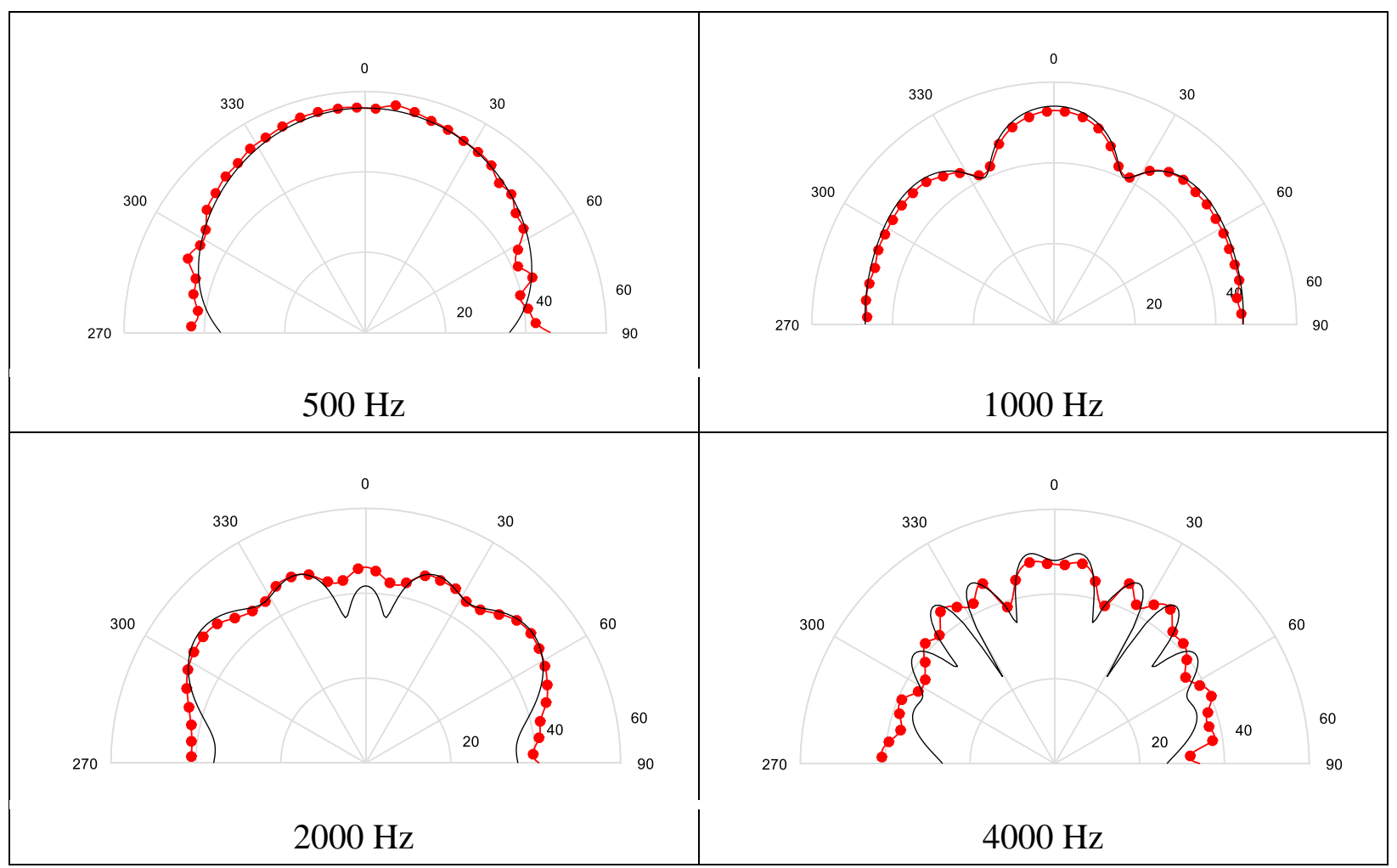

Figure 3: Polar plots in $d B$ (using arbitrary scaling) against angle of incidence in degrees comparing swept-sine measurements (red dotted line) to the numerically modelled predictions (solid black line) of the QRD performance at several frequencies.

The polar responses can be processed using equations 3 and 4 to determine the directional diffusion coefficient, in this case the normal incidence diffusion coefficient (Fig. 4).

A subjective comparison of the measured coefficients to the BEM modelled coefficients indicates that the agreement is reasonable at most frequencies. However, there are four frequencies where the measured diffusion significantly exceeds that predicted (including $2000 \mathrm{~Hz}$ and $4000 \mathrm{~Hz}$, whose polar responses were shown in Fig. 3). In these cases the measured data does not reach the same minima as the modelled response, producing a curve that is closer to semi-circular than predicted, and therefore shows higher diffusion. Of the factors contributing to error in the polar response previously identified, the smoothing of minima is probably mostly due to the band-passed measurements and signal-to-noise ratio, with some contribution from measurement resolution at the highest frequencies. It should also be recognised that the BEM model assumes idealised sound propagation that cannot be realised in real life situations, and so represents something of an unachievable target. We attempted to obtain microphone-based measurements to validate our results, but this was not possible for a single period of the diffusor. Nonetheless, for most frequencies the fit is very good, and for those frequencies where it is not, the causes are evident and involve aspects of the methodology that can feasibly be improved in future. 


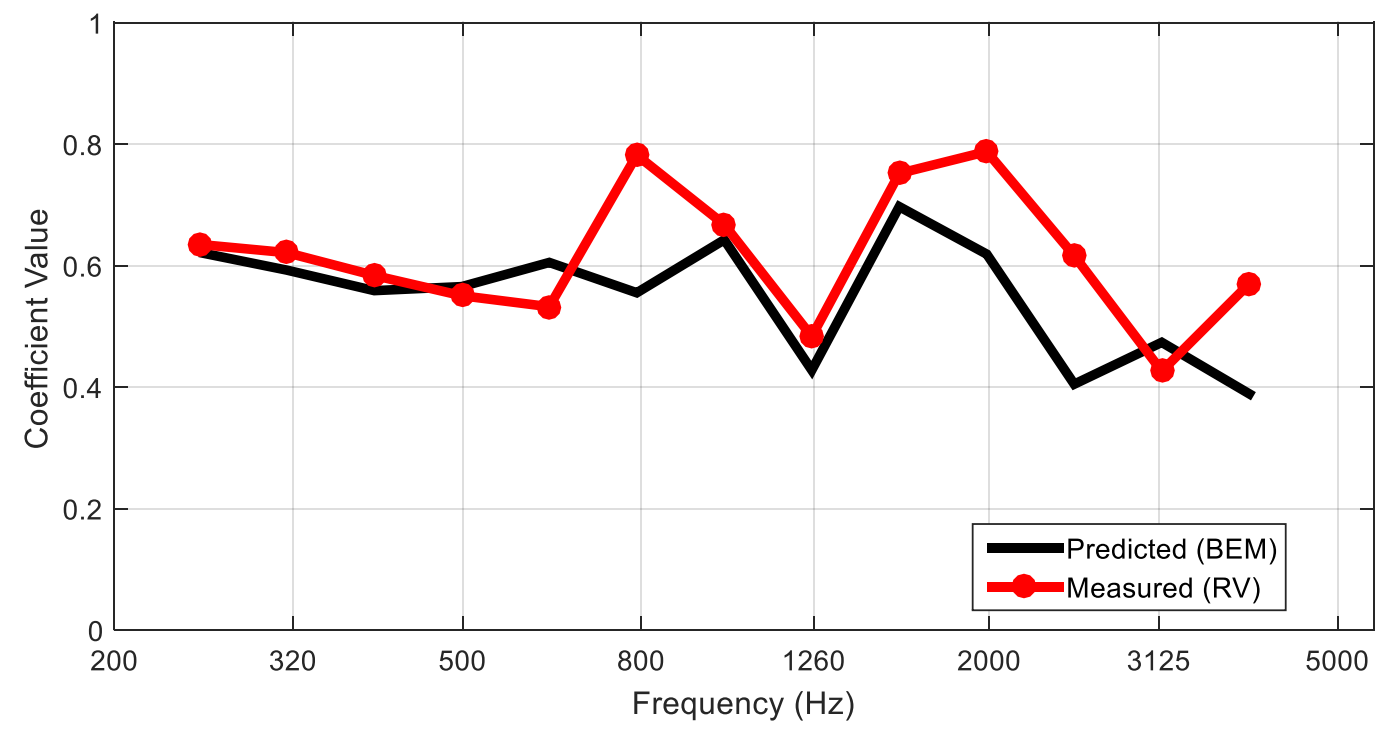

Figure 4: The normal incidence diffusion coefficient as predicted by BEM (black line) and measured by refracto-vibrometry (red dotted line).

\section{DISCUSSION}

These measurements were made at normal incidence, but it is a simple matter to extend the technique to different angles of incidence by rotating the sample and sampling positions, and it follows that random incidence coefficients are straight forward to determine. The approach can be further adapted to determine the directional and random incidence scattering coefficients defined in ISO 17497-1:2004 [4] by way of the correlation scattering coefficient [15], or even modified to find the absorption coefficient [2] by considering the incident sound pressure. It is also possible to examine and quantify the near field performance of the diffuser by setting a smaller radius sampling arc.

The ability of the refracto-vibrometry technique to measure the polar response of the test surface appears to be good, and at a comparable level of precision to microphone based techniques. The authors have not been able to attempt microphone based measurements, and single period data for the QRD-734 has not been available from external sources as usually 3 periods are tested. This highlights a shortcoming of both the optical and microphone approaches to measuring acoustic diffusion, in that a very large space is required to achieve far-field conditions, although in the case of the optical approach a large retroreflector is also required.

In addition to the arc sampling approach described, full field scans with high resolution regular grids can be used either quantitatively (as above), or as animations or freeze-frames to examine the evolution of the reflected sound field (Fig. 1). As a full time series is recorded for every sampling position, it is possible to follow an acoustic perturbation through both time and space to understand its origin and development, and its phase relative to neighbouring waves. Similarly, regions of focusing are very easy to locate with full field scans, as seen in Fig. 1. This kind of measurement would be prohibitively time consuming using a microphone based approach. 
Unlike microphone-based measurements, refracto-vibrometry does not perturb the sound field. This, combined with the option of sub-millimetre 2D spatial resolution and the lack of a physical transducer to move and track, means that refracto-vibrometry is capable of making measurements in situations where traditional approaches would be inappropriate, such as very close to a surface, or directly between source and receiver. On the other hand, refracto-vibrometry requires a clear optical path tangential to the reflecting surface, and so far we have only proven its ability for sampling fields in 2D.

In our experiments, refracto-vibrometry in air has proven sensitive to acoustic frequencies from approximately $500 \mathrm{~Hz}$ to well into the ultrasound range. This makes it ideal for work with scale models, but limits the utility for low frequency work. Sound pressure levels of approximately $90 \mathrm{~dB}$ (re $20 \mu \mathrm{Pa}$ ) or greater are typically required to achieve a reasonable signal-to-noise ratio (significantly higher than would be necessary for microphone based techniques) but recent advances in vibrometer technology are likely to bring this threshold level down significantly.

The vibrometer's sensitivity to pressure changes occurring at any point along the full length of the laser beam has already been discussed in relation to absolute measurement, and remains a limitation to be worked around for most airborne acoustic applications. In some applications it is less of a hindrance, and for measurements to quantify normalised 2D phenomena it can arguably be an asset. However the results should always be considered to be weighted 3D projections onto a 2D plane, rather than a 2D slice through a 3D sound field, as is sometimes the temptation when looking at stills from full field scans.

The measurement approach we used for characterisation of arbitrary reflecting surfaces could also be used to measure the scattering properties of materials. This would be of particular value for materials that have not been accurately modelled (inhomogeneous materials, furs, flexible or moving surfaces), and where microphones would be too coarse or perturbing to use.

\section{CONCLUSION}

The proposed refracto-vibrometry method has proven to be effective at measuring the $2 \mathrm{D}$ polar response of acoustic reflections from an arbitrary anisotropic surface, based on qualitative comparisons against a BEM model for a 1:4 scale QRD. It has also been demonstrated, analytically and by comparison to the BEM model, that the measurements may be used to quantify the directional acoustic diffusion coefficient, and by extension, other normalized quantities including the random incidence diffusion and scattering coefficients.

Refracto-vibrometry in air is still an emerging area of research. Although it has many unexplored applications and enormous potential for further development and refinement, it has so far been employed primarily as a qualitative visualization tool. Quantities of interest to scientists and acousticians, such as sound pressure level at a point, have been elusive because of the difficulty of extracting point data from the line integral inherent to the approach. However, determining dimensionless quantities such as the coefficients describing reflection in a plane does not require an absolute measurement. Leveraging this fact, a new measurement and analysis process relying on refracto-vibrometry has been developed and used to calculate the acoustic diffusion coefficient through purely optical means for the first time. 


\section{ACKNOWLEDGEMENTS}

The authors acknowledge the assistance of their colleagues at the National Physical Laboratory, in particular Peter Woolliams, and of the facilities and equipment maintained through funding from the National Measurement and Regulation Office of the UK Department for Business, Energy \& Industrial Strategy. The authors are very grateful for data and advice provided by Dr. Peter D'Antonio (RPG Diffusor Systems) and Dr. Trevor Cox (University of Salford).

\section{REFERENCES}

[1] W.C. Sabine, Collected Papers on Acoustics, Harvard University Press, Cambridge Mass., 1923, pp. 3-68, 69-105, 163-197.

[2] British Standards Institution, BS EN 12354-6, Building acoustics. Estimation of acoustic performance of buildings from the performance of elements. Sound absorption in enclosed spaces, 2003.

[3] International Organization for Standardization ISO 17497-2, Acoustics. Sound-scattering properties of surfaces. Measurement of the directional diffusion coefficient in a free field., 2012. [4] International Organization for Standardization ISO 17497-1, Acoustics. Sound-scattering properties of surfaces. Measurement of the random-incidence scattering coefficient in a reverberation room, 2004.

[5] J. Rienitz, Schlieren Experiments 300 years ago. Nature 254 (1975) 293-295.

[6] A. Torras-Rosell, S. Barrera-Figueroa, F. Jacobsen, Sound field reconstruction using acousto-optic tomography. Journal of the Acoustical Society of America 131 (2012) 3786-3793. [7] C.B. Scruby, L.E. Drain, Laser Ultrasonics and Applications, Taylor \& Francis Group, New York, 1990, pp. 37-46, 76-146.

[8] P. Theobald, R. Lord, W. Yuebing, Mapping of scattered acoustic fields in air using the acousto optic effect, Proceedings of the Spring Conference of the Institute of Acoustics, Southampton, 2006, pp. 493-498.

[9] B. Cook, Ultrasonic field determination by a form of computer-aided tomography applied to acousto-optic data. Journal of the Acoustical Society of America 62, S21 (1977).

[10] A. Torras-Rosell, S. Barrera-Figueroa, A new method to characterize impulsive sources using acousto-optic interaction, Proceedings of Internoise, San Francisco, 2015.

[11] M.R. Schroeder, Binaural Dissimilarity and Optimum Ceilings for Concert Halls: More Lateral Sound Diffusion. Journal of the Acoustical Society of America 65 (1979) 958-63. 
[12] QRD-734 Specification Sheet, RPG Acoustics, http://www.rpginc.com/ProductDocs/QRD7_QRD 734/QRD 734_Acoustical Data.pdf (Accessed 12 August 2016).

[13] R. Jackett, B. Piper, Characterization of acoustic diffusion using refracto-vibrometry, Proceedings of Internoise, San Francisco, 2015.

[14] T.J. Cox, Predicting the scattering from reflectors and diffusers using two-dimensional boundary element methods. Journal of the Acoustical Society of America 96 (1994) 874-878. [15] T.J. Cox, B-I.L. Dalenback, P. D’Antonio, J.J. Embrechts, J.Y. Jeon, E. Mommertz, M. Vorlander, A Tutorial on Scattering and Diffusion Coefficients for Room Acoustic Surfaces. Acta Acustica 92 (2006) 1-15.

(c) Queen's Printer and Controller of HMSO, 2016 